\title{
The Role of Urodynamic Investigations in Management of Stress Urinary Incontinence
}

\author{
Ghirca Veronica Maria*, Porav-Hodade D, Chibelean C, Voidazan S, Vartolomei M, Boja R, Martha \\ Orsolya
}

University of Medicine and Pharmacy Tîrgu Mureș, Romania

\begin{abstract}
Objective: The aim of this study is to establish the importance of urodynamic investigations in women diagnosed with stress urinary incontinence (SUI) who have indication of surgical treatment. Methods: We performed a retrospective study over a period of 3 years (January 2012-December 2014) in Clinic of Urology from Tirgu Mures. The inclusion criteria were: female patients diagnosed with SUI having indication of surgical treatment and the existence of urodynamic investigations (uroflowmetry and pressure-flow study). We evaluated 118 patients with SUI. From this patients, 24 cases (20.3\%) accomplished the criteria from above. Results: We included in this study 24 patients aged $64.25+/-8.25$ (standard deviation). Pressure-flow study revealed an impaired detrusor contraction in 13 cases. Statistical anaysis pouved a relation between existence of post void residual urine (PVR) and underactive detrusor (UD) $(p=0.01)$. There is no correlation between maximum flow rate $(Q \max )$ and $U D, r=0.18(C l=-0.2-0.5), p=0.3$ and between normal value of $Q m a x$ and normal detrusor pressure $(P d e t), r=0,28(C l=-$ 0.6-0.8), $p=0.58$. Also there is no relation between a low $Q \max$ and UD, $p=0,5$. There is a statistical relation between increased abdominal pressure (Pabd) and UD, $\mathrm{p}=0.02$. Conclusions: Uroflowmetry has the role to guide us concerning the micturition process Pressure-flow study is indicated in management of SUI, in selected cases, in patients with voiding symptomatology, the suspicion of a detrusor contractility dysfunction, abnormal uroflowmetry results, existence of PVR, in prediction of the surgical treatment outcome or if we think that the findings can change the choice of treatment.
\end{abstract}

Keywords: post-void residual urine, pressure-flow study, stress urinary incontinence, Urodynamics, uroflowmetry

Received: 26 July 2015 / Accepted: 03 September 2015

\section{Introduction}

Stress urinary incontinence (SUI) according to the ICS, represents the involuntary loss of urine from the urethra on effort, exertion, sneezing or coughing in the absence of involuntary detrusor contraction [9].

This condition, having a negative impact on the patient's quality of life, provokes an increasing interest concerning the diagnosis and treatment possibilities.

It has been estimated that urinary incontinence in women is the reason why more than 1.1 million of patients visited the doctor's office in 2000, in United States (Litwin et al, 2005).

Current studies show a dispersed prevalence of urinary incontinence among the population. About $10 \%$ of adult females report weekly urinary loss and between 25\%-45\% of these report occasional loss of urine [8].

The prevalence of the disease varies depending on the geographical area or race. EpiLUTS study showed a similar prevalence in United States, Britain and Sweden, (approximately $29.5 \%$ ), while in Italy this was lower (9.3\%). In the case of asian women the prevalence of urinary incontinence is quite low.

Also there is a significant difference between the existence of stress urinary incontinence between afro-american women compared to caucasian one, in the favour of afroamericans who present a twice lower incidence [8].
Among the diagnostic tools, the urodynamic investigations may be useful, together with the clinical examination in order to confirm the diagnosis. These procedures can provide real informations regarding the type, grade of urinary incontinence and the provocative factors, manouvers of urine loss and can influence the way of treatment without affecting the patient's evolution.

The European Guidelines (2015) recommend urodynamic evaluation in the management of SUI if the findings will change the choice of an invasive treatment.

The aim of this study is to establish the importance of urodynamic investigations in the diagnostic protocol of stress urinary incontinence with indication of surgical management.

\section{Methods}

We performed a retrospective study over a period of 3 years (January 2012-December 2014) in Mures County Hospital, Clinic of Urology.

In the mentioned period we evaluated 118 patients with stress urinary incontinence. 34 of them $(28.8 \%)$ with surgical indication. From this group of 34 patients, 24 (20.3\%) accomplished the inclusion criteria, so were included in the study and the findings were statisticaly analyzed .

The selection criteria for this study were the following:

- Female patients suffering from stress urinary incontinence proved by urodynamic investigations with indication of surgical treatment. 
- Pressure-flow studies were indicated carefully in cases of patients who had one or more of the following conditions: voiding symptoms, diabetes for many years, history of obstetric or pelvic surgery, history of cerebrovascular disease, stress urinary incontinence surgery, advanced age, abnormal uroflowmetry result, post-void residual urine (PVR).

The investigation protocol used for the selected cases consisted of:

- History: questionnaires (ICIQ-UI), details regarding type and severity of urinary incontinence, the existence of other conditions like diabetes, neurological pathology, history of cerebrovascular disease, constipation, depression, sexual activity, obstetric history (number of births, labor and vaginal delivery, prolapse symptoms or surgery, hysterectomy), medication, associated pathology for urinary incontinence or impaired detrusor contractility.

- Micturition diary to quantify the micturition frequency during day and night, the voided volume frequence and grade of urine loss.

- Clinical examination (abdominal, perineal, vaginal and rectal examination)

- Abdominal ultrasound including post-void residual urine determination

- Urinanalysis and bacteriological examination to identify an urinary tract infection. In case of positive findings an antibiotic treatment was initiated preoperatively in concordance to the bacteriological sensitivity

- Urodynamic investigations (uroflowmetry and pressure-flow study)

Concerning the urodynamic investigations first we performed uroflowmetry to all the patients included in the study.

According to the patient's history and associated risk factors, diabetes, advanced age, neurogenic disease, obstetric history or investigations that sugested a detrusor contractility dysfunction, we performed also preoperative pressure-flow study to all the patients selected in the study. This investigation was useful for the accuracy of the diagnosis (including the detrusor contraction), indication of surgical management of SUI and prediction of a favourable result of the treatment.

Statistical analysis was performed using the MedCalc Software, Version 12.5.0.0. Data were considered as nominal or quantitative variables. Nominal variables were characterized using frequencies. Quantitative variables were tested for normality of distribution using KolmogorovSmirnov test and were characterized by median and percentiles (25-75\%) or by mean and standard deviation (SD), when appropriate. A chi-square test was used in order to compare the frequencies of nominal variables. Quantitative variables were compared using $t$ test, Mann-Whitney test. The correlation between quantitative variables was assessed using Spearman's rho, when appropriate. Multivariate analysis was carried out using linear regressions. We used as dependent variable the stress urinary incontinence. We included as independent variables the data for diabetes and neurological deseases, pelvic surgery . The level of statistical significance was set at $\mathrm{p}<0.05$.

\section{Results}

In a period of 3 years (January 2012- December 2014) in the Clinic of Urology from Tg. Mures were diagnosed and treated 118 patients with SUI. From the total of 118 women presenting stress urinary incontinence $(100 \%)$, we evaluated in our study, $24(20.2 \%)$ patients who accomplished the inclusion criteria.

Majority of the patients were from the group aged 6069 years (12 cases), followed by 70-79 years (6 cases), 5059 years ( 4 cases) and $40-49$ years $(2$ cases). The mean age

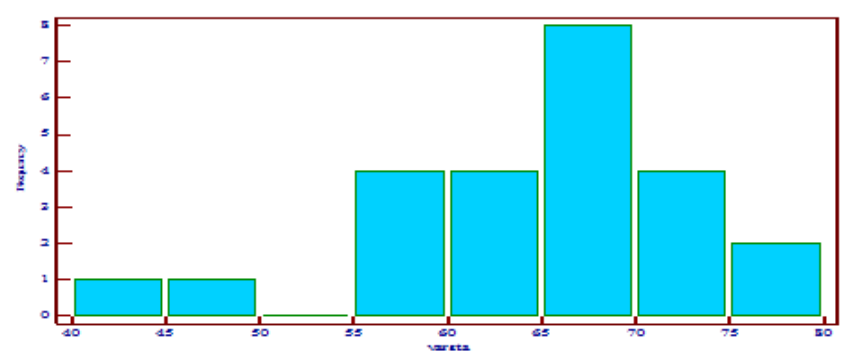

Fig.1. Age prevalence of SUI

was $64.25+/-8.25$ (standard deviation) years old with extremities between 41 to 76 years (Figure 1).

Majority of the patients reported between 1-4 pregnances (22 cases), 1-3 vaginal delivery in 19 cases and caesarian in 6 cases. Pathological history and clinical examination established the existence of menopause in 22 cases, genital prolapse in 7 cases, diabetes in 12 cases, history of cerebrovascular disease in 5 cases, obstetric surgical history (partial or radical hysterectomy) in 9 cases, history of repeated urinary tract infections in 9 cases.

In 7 of the cases we observed post void residual urine (PVR) ( $>50 \mathrm{ml}$ ) objectified by transabdominal ultrasound. The median value of post void residual urine was $80 \mathrm{ml} /$ 48.99 (standard deviation) with wider extremities between $50 \mathrm{ml}$ and $190 \mathrm{ml}$.

Uroflowmetry was performed to all of the patients included in the study. The arithmetic mean of maximum flow rate (Qmax) was $16.95 \mathrm{ml} / \mathrm{s}+/-7.8$ (standard deviation) with the extremities between $2 \mathrm{ml} / \mathrm{s}$ and $33 \mathrm{ml} / \mathrm{s}$. We observed in 15 of the cases, a prolonged or asymmetrical curve and extended flow time. The arithmetic mean of Qmax in these cases was $11.93 \mathrm{ml} / \mathrm{s}+/-3.73$ (standard deviation) with extremities between $2 \mathrm{ml} / \mathrm{s}$ and $15 \mathrm{ml} / \mathrm{s}$.

Pressure -flow study revealed an impaired detrusor contraction in cases of 13 women and normal contraction in 11 cases.

In the filling phase of the pressure flow study, we observed loss of urine in different quantities. The mediane value was $50 \mathrm{ml}$, the arithmetic mean was $55.8 \mathrm{ml}+/-41.6$ (standard deviation) with values between 20 and $182 \mathrm{ml}$. 
Regarding the bladder capacity, 6 of the patients had a high one (over $400 \mathrm{ml}$ ) while 18 of them had a normal capacity. We applied Chi-square test which revealed no relation between the bladder capacity and diagnosis of underactive detrusor $(\mathrm{p}=0.8)$.

In the voiding phase of pressure-flow study, the detrusor presure (Pdet) had a mean value in "normal" cases of $27.73+/-6.5$ (standard deviation) compared with the value of Pdet in cases with underactive detrusor that was lower, $11.46+/-4.7$ (standard deviation). We applied Unpaired $\mathrm{t}$ test that revealed a strong relation between low pdet and the existence of underactive detrusor $(\mathrm{p}=0.0001)$.

We applied Chi-square test and we calculated the relation between diabetes and the existence of underactive detrusor, value of $\mathrm{p}$ was insignificant $(\mathrm{p}=0.4)$.

We evaluated also the relation between the existence of underactive detrusor and history of neurological disease (stroke), diabetes and obstetric surgery (partial or radical hysterectomy). We calculated the logistic regression and we observed that underactive detrusor is dependent influenced by the obstetric surgeries, $p$ was significant $(\mathrm{p}=0.05)$. Odds radio value was 7.2 with a confidence interval between 1.8 to 57.9 , so that patients with history of obstetric surgery have 7.2 times risk to develop underactive detrusor than patients without surgery.

Concerning the relation between the existence of postvoid residual urine and detrusor contraction. Mann-Whitney test revealed that there is a statistical relation between the existence of post void residual urine and underactive detrusor $(\mathrm{p}=0.01)$. All the patients with post void residual urine were diagnosed with underactive detrusor after performing pressure-flow study.

From statistical point of view we were interested if there is any correlation between the value of Qmax and pressure of detrusor (Pdet) suggestive for underactive detrusor, so that we calculated the correlation coefficient, $r=0.18$ (confidence interval for $r=-0.2-0.5)$ which revealed that there is no correlation between them, also value of $p$ was insignificant $(p=0.3)$. Also we applied Unpaired $t$ test which revealed that there is no relation between low Qmax and low Pdet, $(\mathrm{p}=0.5)$. Also, we calculated the correlation coefficient between normal value of $\mathrm{Qmax}$ and the existence of normal Pdet and we observed that there is no correlation $\mathrm{r}=0.28$ (confidence interval for $\mathrm{r}=-0.6-0.8$ ), $\mathrm{p}$ value was insignificant, $\mathrm{p}=0.58$.

In 9 of the cases we observed an elevated abdominal pressure (pabd) in voiding phase, while in 15 of the cases Pabd was normal. We applied Chi-square test that revealed a statistical relation between the increasing Pabd and the existence of underactive detrusor $(\mathrm{p}=0.02)$.

\section{Discussion}

Stress urinary incontinence is a very frequent disease, which has a negative impact of women's quality of life. Most of the patients reported the absence of sexual activity, the loss of urine affects their social and professional activ- ity, daily confort and hygiene. A proper management and a personalized treatment is mandatory. In the management of SUI there is no need to perform pressure-flow study to all of the patients, especially in cases of conservative treatment modalities.

Invasive urodynamic investigations can be performed whenever surgical intervention is planned, whenever there is doubt about the pathophysiology, or about whether the UI is uncomplicated or not in order to provide the treatment decision and prognosis [8].

Our study, performed in cases of 118 patients presenting SUI, revealed that the predominant symptom was loss of urine during effort, coughing, laughing. In 24 of the cases $(20.2 \%)$ patients related voiding symptoms revealed by the existence of post void residual urine in 7 cases, abnormal uroflowmetry curve and parameters (15 cases) or the past history of other associated diseases like diabetes (12 cases), stroke (5 cases), obstetric surgeries like partial or radical hysterectomy (9 cases), urinary tract infections ( 9 cases), genital prolapse (7 cases) and others. In these cases we performed pressure-flow study. The role of urodynamic investigation is to translate the sign and/or symptoms into a clinical diagnosis and a proper way of treatment [8].

Also, we observed that most of the patients were aged 60 or above (12 cases). Older women with SUI can have worsened voiding function with age, especially a decreased detrusor contractility [7]. In our study 9 of the patients diagnosed with detrusor underactivity were aged 60 or above.

In spite of urine loss, in 7 cases from the total of $24 \mathrm{pa}-$ tients, we detected post-void residual urine (PVR) evaluated after performing abdominal ultrasoud. The mean value of PVR was $80+/-48.99$ (standard deviation).

After performing uroflowmetry, from the total of 24 cases, in 9 the curve was normal, but in 15 cases we observed a prolonged or interrupted curve, with a low maximum flow rate $(\mathrm{Qmax})$ value between $2-15 \mathrm{ml} / \mathrm{s}$. Abnormal flow rates, may be useful in predicting post-operative voiding dysfunction and retention following surgical treatment of SUI [8].

Urodynamic practice demandes that abdominal pressure (Pabd), intravesical pressure (Pves) as well as detrusor pressure (Pdet) are evaluated to diagnose urinary bladder function [8].

Pressure-flow study revealed in the filling phase that bladder sensations were normal and the loss of urine was between 20-189 $\mathrm{ml}$ with a median of $50 \mathrm{ml}$.

In most of the cases the bladder capacity was normal (18 cases), but in 6 cases the capacity was more than 400 $\mathrm{ml}$, in 3 of these cases pressure-flow study proved a normal detrusor contraction and in 3 of them the condition was associated with underactive detrusor. High bladder capacity ( more than $400 \mathrm{ml}$ ) has no relation with the existence of underactive detrusor $(\mathrm{p}=0.8)$.

Voiding phase of the pressure-flow studies revealed from the total of 24 cases, an impaired detrusor contraction (transposed by the low detrusor pressure value) In 13 cases and normal contraction in 11 cases. 
Following we analyzed the relation between PVR, the results of uroflowmetry and pressure-flow study.

The uroflowmetry performed in the 7 cases with PVR, revealed a prolonged curve with a low Q max (under 15 $\mathrm{ml} / \mathrm{s}$ ) in 6 cases and an abnormal curve, interrupted but with a normal Qmax in 1 case. Voiding phase evidenced a low Pdet pressure in all the cases. In 2 cases genital prolapse gr. III/IV was associated, which proved to be obstructive with low Qmax value. The case with normal Qmax was due to abdominal effort during the micturition. The statistical analysis prouved a strong relation between the existence of post void residual urine and underactive detrusor $(\mathrm{p}=0.01)$ in these cases. An underactive detrusor confirmed by pressure-flow study doesn't lead in all of the cases to the appearance of post-void residual urine.

Concerning the uroflowmetry results, from the total of 24 patients in 15 cases we observed a prolonged or interrupted curve, with a low Qmax value. Patients with poor flow rates or abnormal pressure-flow result before surgery, are informed that they have a higher risk of having voiding problems following surgical treatment of SUI (UI). In our study, 11 of these cases were diagnosed with underactive detrusor after performing pressure-flow study and in 3 cases the low Qmax value was due to obstructive causes. In 2 of the cases diagnosed with underactive detrusor, Qmax was normal due to the abdominal effort during micturition. This hypothesis was confirmed after performing pressure-flow study. We had 1 case with genital prolapse gr III/IV and low Qmax caused by the obstruction objectified after performing pressure-flow study (high Pdet). In 2 cases, genital prolapse was associated with underactive detrusor. Qmax had a low value, but pressure-flow study investigation revealed the diagnosis.

Statistical analysis proved that there is no relation between a low Qmax and existence of underactive detrusor $(\mathrm{p}=0.3)$. Also, there is no correlation between the normal value of $\mathrm{Qmax}$ and normal pressure of detrusor $(\mathrm{p}=0.58)$, so that uroflowmetry doesn't provide enough informations concerning the detrusor contractility. In this cases is important to practice pressure-flow study when we decide to perform a surgical treatment for SUI. This investigation will differentiat a low Qmax caused by an obstruction or an impaired detrusor contractility and on the other side will differentiate a normal Qmax with a normal contraction by a normal $\mathrm{Q}$ max value due to the effort during micturition. A low Qmax doesn't necessary represent a detrusor contractility dysfunction.

From this point of view is important to analyse the abdominal pressure (Pabd) during voiding phase of pressureflow study.

In 15 of the cases, the abdominal pressure was normal, but in 9 of them Pabd was increased. 8 of the cases with high Pabd were associated with a low Pdet. In 1 case with genital prolapse gr III/IV, Pabd was high secondary to the obstruction. Statistical analysis proved that there is a relation between increased Pabd, that represent an abdominal effort during micturition and existence of underactive detrusor $(\mathrm{p}=0.02)$.

Also, we found that in our study it was no relation between diabetes and the existence of underactive detrusor $(p=0.4) .8$ cases with diabetes were diagnosed with underactive detrusor. According to our results, this condition was influenced by the obstetric surgeries in the past history.

\section{Conclusions}

Urodynamic investigations are very important in the diagnosis management of SUI.

Uroflowmetry can be performed to all the patients due to it's noninvasive character. It was the role to guide us concerning the micturition process. A low $\mathrm{Qmax}$ value does not necessarily mean a low detrusor contraction ans a normal Qmax value does not exclude a detrusor contractility dysfunction, so that we can not use the uroflowmetry to predict the detrusor function. In this case the shape of the uroflowmetry curve associated with proper history answers, and clinical examination can guide the diagnosis.

In most of the cases the existence of post-void residual urine can indicate a detrusor contractility dysfunction that can compromise the result of our therapy.

Also, pressure-flow study (voiding phase) is important in differentiateing a low Qmax caused by an obstruction by a low Qmax secondary to a detrusor contractility dysfunction. The evaluation of Pdet in voiding phase is mandatory.

Pre-operative pressure-flow study investigation are useful for the accuracy of the diagnosis, indication of surgical managent os SUI and the prediction of a favourable result of the treatment.

Anyway, we belive that pressure-flow study is indicated in management of SUI, in selected cases, in patients with voiding symptomatology, when we suspect the existence of a detrusor contractility dysfunction, in cases with abnormal uroflowmetry result, existence of post-void residual urine, the prediction of the surgical treatment outcome or if we think that the findings can change the choice of treatment

\section{Acknowledgement}

This paper was published under the frame of European Social Found, Human Resources Development Operational Programme 2007-2013, project no. POSDRU/159/1.5/S/133377".

\section{References}

1. G. Bumbu, Orsolya Martha. Incontinenta urinara. Tratat de urologie. 2007;44:3007-3032

2. Orsolya Martha. Tratamentul conservator in incontinenta urinara de efort (IUE) la femei (reabilitarea aparatului urinar inferior). Tratat de urologie. 2007;44:3033-3037

3. M.G. Lukas, D. Bedretdinova, J.L.H.R. Bosch, et al. Urinary incontinence. Guidelines 2014;30-50;73-94

4. Hermieu JF. Recommendations for the urodynamic examination in the investigation of non-neurological female urinary incontinence. Prog Urol. 2007;17(6Suppl2):1264-84. 
5. C.R. Chapple, S.A. MacDiarmid, A. Patel. Urodynamics made easy, Storage disorders and incontinence. Third edition. 2009;103-127.

6. Elizabeth A. Miller, Cindy L. Amundsen, Khai Lee Toh, B. J. Flyn, G. D. Webster, Preoperative Urodynamic Evaluation May Predict Voiding Dysfunction in Women Undergoing Pubovaginal Sling. Department of Urology. 2003
7. Shin YS, On JW, Kim MK. Effect of aging on urodynamic parameters in women with stress urinary incontinence. Korean J Urol. 2015;56(5):393-7

8. P. Abrams, L. Cardozo, S. Khoury, A. Wein. Incontinence. 5th Edition. 2013;432-461

9. Campbell-Walsh Urology. Urinary incontinence and pelvic prolapse: epidemiology and pathophysiology, tenth editin. 2010;1871-1882 